\title{
Programming Directional Deep Brain Stimulation in Parkinson's Disease: A Randomized Prospective Trial Comparing Early versus Delayed Stimulation Steering
}

\author{
Ricardo Maciel $^{\mathrm{a}}$ Derrick Soh ${ }^{\mathrm{a}, \mathrm{b}}$ Renato P. Munhoz ${ }^{\mathrm{a}}$ Yu-Yan Poon ${ }^{\mathrm{a}}$ \\ Suneil K. Kalia ${ }^{c}$ Mojgan Hodaie ${ }^{c}$ Andres M. Lozano ${ }^{c, d}$ Alfonso Fasano a, d,e \\ aEdmond J. Safra Program in Parkinson's Disease and the Morton and Gloria Shulman Movement Disorders Centre, \\ Toronto Western Hospital, UHN, Toronto, ON, Canada; 'beurology Department, The Alfred Hospital, Melbourne, \\ VIC, Australia; 'Division of Neurosurgery, Department of Neurosurgery, Toronto Western Hospital, University \\ of Toronto, Toronto, ON, Canada; ${ }^{\mathrm{d} K r e m b i l}$ Brain Institute, Toronto, ON, Canada; ${ }^{\mathrm{e} T h e}$ Center for Advancing \\ Neurotechnological Innovation to Application (CRANIA), Toronto, ON, Canada
}

\author{
Keywords \\ Deep brain stimulation - Directional leads · Parkinson's \\ disease $\cdot$ Programming
}

\begin{abstract}
Introduction: Programming directional leads poses new challenges as the optimal strategy is yet to be established. We designed a randomized control study to establish an evidence-based programming algorithm for patients with Parkinson's disease undergoing subthalamic nucleus deep brain stimulation with directional leads. Methods: Fourteen consecutive patients were randomized to programming with either early or delayed (i.e., starting with a "ring mode") steered stimulation. Motor scores, number of programming visits, calls to the clinic, battery consumption, and stimulation adjustments required were recorded and compared between groups, using the Wilcoxon signed-ranks test, after 3 months of open-label programming. Results: Thirteen patients ( 25 electrodes) were included, of which 23 were steerable. Nine out of 14 electrodes allocated to delayed steered stimulation were changed to steered mode due to side effects during the course of the study. No patients ( 11 electrodes) initially allocated to early steered stimulation were
\end{abstract}

karger@karger.com www.karger.com/sfn

Karger $\stackrel{\text { ' }}{5}$

BOPEN ACCESS
(C) 2021 The Author(s)

Published by S. Karger AG, Basel

This is an Open Access article licensed under the Creative Commons Attribution-NonCommercial-4.0 International License (CC BY-NC) (http://www.karger.com/Services/OpenAccessLicense), applicable to the online version of the article only. Usage and distribution for commercial purposes requires written permission. converted to ring mode. The 2 study arms did not differ in any of the considered measures at 3 months. Conclusion: Programming with early or delayed steered stimulation is equally effective in the short term. However, delayed steering is less time consuming and is not always needed.

(c) 2021 The Author(s).

Published by S. Karger AG, Basel

\section{Introduction}

Newer deep brain stimulation (DBS) electrodes with radially segmented contacts allow for shaping of the stimulation field in the plane orthogonal to the lead, providing added flexibility to programming. Indeed, steered stimulation has been found to expand the therapeutic window (TW) in Parkinson's disease (PD) $[1,2]$.

The last literature-based programming algorithms published did not contain recommendations for directional lead programming [3]. As technology advances, the added complexity of programming can become daunting as the number of different programming op-

Ricardo Maciel and Derrick Soh have contributed equally.
Correspondence to:

Alfonso Fasano, alfonso.fasano@uhn.ca 


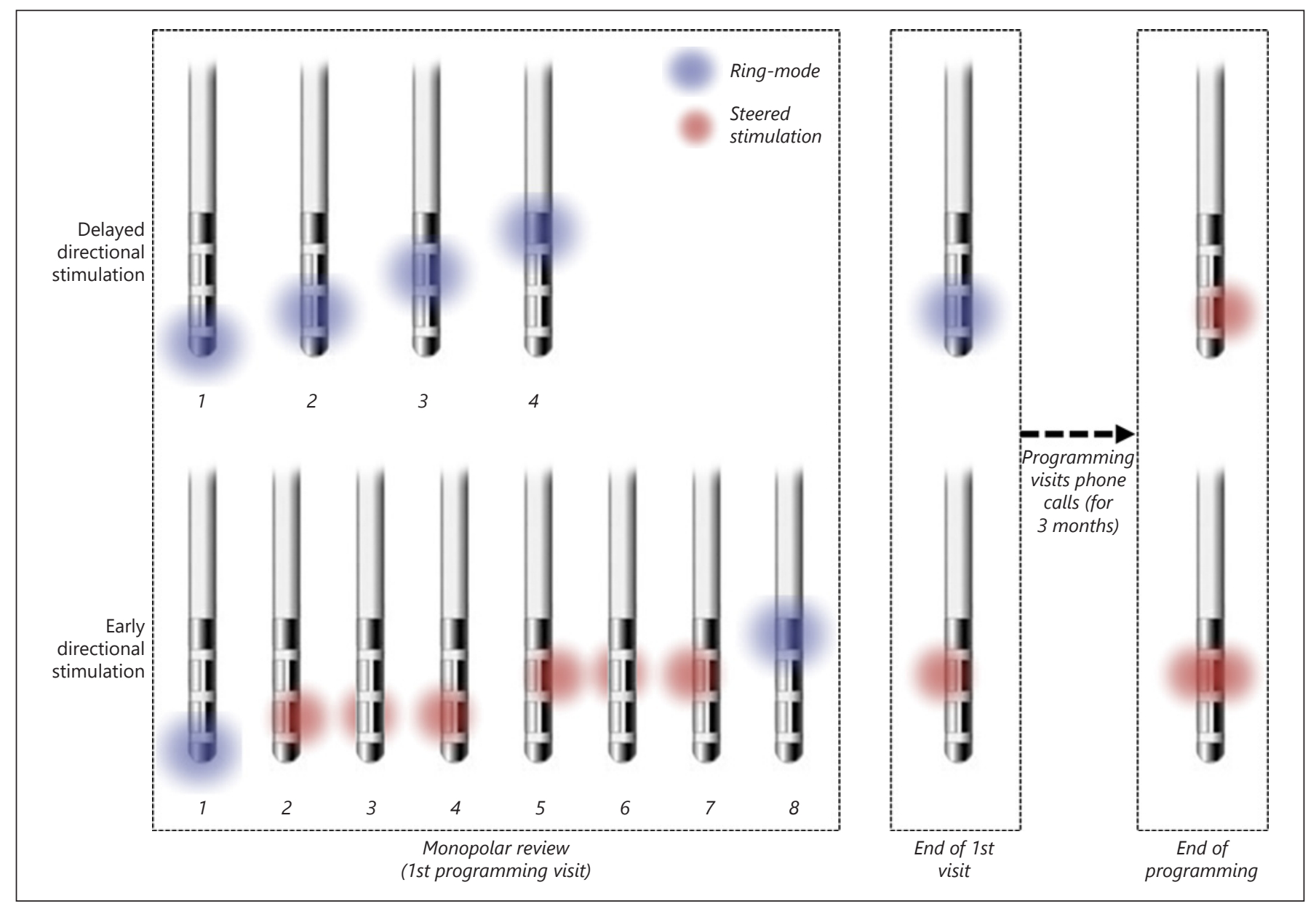

Fig. 1. MR and initial programming by group allocation. The MR was performed level by level (4 rings) or segment by segment ( 2 rings plus 6 segments) according to group allocation. MR, monopolar review.

tions increases exponentially. This in turn might increase patients' and physicians' expectations and frustration [4].

The first step in DBS programming is the monopolar review (MR), in which the contact with the best TW is selected for stimulation. When only nonsteered leads were available, MR was a level-by-level review of 4-8 different vertical levels [3]. The currently available steered leads feature 8 contacts for 4 levels (as the 2 middle levels are split into 3 contacts each) resulting in a more complex MR. There are 2 options for the MR of steered leads: a proper MR (8 conditions per side) or a level-by-level review using all levels in ring mode (4 conditions per side).

There are no studies comparing the effectiveness and health care burden (i.e., visit length, frequency of visits and other contacts with the service, and stimulation changes other than increase in amplitude) of programming strategies for steered leads. We present the results of the first prospective randomized trial aiming at establishing the best programming algorithm for directional DBS.

\section{Methods}

Fourteen consecutive PD patients who had undergone subthalamic nucleus (STN) DBS with steered leads were recruited from June 2018 to February 2019. Inclusion and exclusion criteria for DBS followed previous published criteria [5]. All participants were implanted with a Vercise Cartesia ${ }^{\mathrm{TM}}$ system (Boston Scientific, Marlborough, MA, USA) connected to a rechargeable implanted pulse generator (Vercise Gevia ${ }^{\mathrm{TM}}$ ) according to standards of care. Conscious sedation and microelectrode recordings were used in all patients for electrode placement. Patients then underwent programming 1 month after surgery and were assigned using block randomization to either of the following (shown in Fig. 1):

- Delayed steered stimulation (DSS) (i.e., standard programming): an MR was performed level by level (4 per side) in "ring 
Table 1. Baseline features and DBS outcomes according to the initial programming strategy

\begin{tabular}{|c|c|c|c|}
\hline & DSS & ESS & $p$ value \\
\hline \multicolumn{4}{|l|}{ Baseline } \\
\hline Patients, $n$ & 7 & 7 & na \\
\hline Sex $(M: F)$ & $6: 1$ & $6: 1$ & 1.0 \\
\hline Age, years & $60.0 \pm 9.1$ & $64.4 \pm 5.5$ & 0.29 \\
\hline MDS-UPDRS I & $3.5 \pm 1.5$ & $3.3 \pm 0.6$ & 0.86 \\
\hline MDS-UPDRS II & $19.8 \pm 7.2$ & $15.0 \pm 2.4$ & 0.11 \\
\hline MDS-UPDRS-III med off & $37.9 \pm 6.9$ & $40.6 \pm 3.7$ & 0.38 \\
\hline MDS-UPDRS-III med on & $11.1 \pm 5.7$ & $15.7 \pm 6.9$ & 0.20 \\
\hline MDS-UPDRS-IV & $7.0 \pm 3.1$ & $6.8 \pm 3.2$ & 0.92 \\
\hline LEDD, mg/day & $1,588 \pm 394.8$ & $1,667 \pm 542.7$ & 0.76 \\
\hline $\mathrm{MoCA}$ & $28.1 \pm 1.3$ & $26.2 \pm 4.1$ & 0.27 \\
\hline \multicolumn{4}{|l|}{3 months after DBS } \\
\hline Patients included in the analysis & 7 & $6^{*}$ & \\
\hline Steerable/total leads & $13 / 14(92.9 \%)$ & $10 / 11^{*}(90.1 \%)$ & 0.90 \\
\hline Change to steered (for DSS)/ring (for ISS), $n(\%)$ & $9 / 13(69.2)$ & $0 / 10(0)$ & 0.001 \\
\hline MDS-UPDRS-III med off & $24.14 \pm 11.51$ & $33.33 \pm 6.62$ & 0.11 \\
\hline MDS-UPDRS-III med on & $18.20 \pm 9.65$ & $23.00 \pm 3.74$ & 0.38 \\
\hline Change in MDS-UPDRS III off & $-13.76 \pm 3.48$ & $-7.27 \pm 3.76$ & 0.18 \\
\hline MDS-UPDRS-IV & $4.42 \pm 3.55$ & $2.67 \pm 2.33$ & 0.32 \\
\hline Change in MDS-UPDRS IV & $-2.51 \pm 1.17$ & $-4.23 \pm 1.26$ & 0.34 \\
\hline $\mathrm{LEDD}, \mathrm{mg} /$ day & $981.29 \pm 450.07$ & $765.00 \pm 418.76$ & 0.39 \\
\hline Change in LEDD & $-620.22 \pm 154.99$ & $-740.49 \pm 154.99$ & 0.59 \\
\hline Visits, $n^{* *}$ & $7.5(7.0-8.25)$ & $7.0(6.0-8.0)$ & 0.20 \\
\hline Calls, $n^{* *}$ & $0.5(0.0-4.0)$ & $2.0(1.0-4.0)$ & 0.34 \\
\hline Contact adjustments, $n^{* *}$ & $1.5(0.75-2.25)$ & $2.0(1.0-2.0)$ & 0.73 \\
\hline Other adjustments, $n^{* *}$ & $0.5(0.0-1.0)$ & $0.0(0.0-2.0)$ & 1.0 \\
\hline Battery recharge time & $1 \mathrm{~h} /$ week & $1 \mathrm{~h} /$ week & \\
\hline
\end{tabular}

DBS, deep brain stimulation; DSS, delayed steered stimulation, F, females; ESS, early steered stimulation; IQR, interquartile range 25-75\%; LEDD, levodopa equivalent daily dose; M, males; MDS-UPDRS, Movement Disorders Society United Parkinson's Disease Rating Scale; MoCA, Montreal Cognitive Assessment. Bold-typed values represent statistically significant differences. ${ }^{*}$ One patient with unilateral DBS and 1 patient dropped out after surgery due to severe dysphagia. ${ }^{* *}$ Median (IQR).

mode" in keeping with published guidelines [3]; the level with best TW was chosen for initial stimulation, and current was steered only during following programming visits as required.

- Early steered stimulation (ESS): an MR was performed contact by contact ( 8 per side); the contact with the best TW was chosen for initial stimulation, and current was increased (as well as volume of stimulation by adding more contacts) during following programming visits as required.

Patients completed 5 weekly visits of open-label programming in keeping with standards of care and were reviewed 3 months after, with additional visits as needed. During these visits, stimulation was optimized by changing parameters or by adding or removing contacts. Steered stimulation was allowed irrespective of initial group allocation. When changing from ring to steered stimulation, stimulation was moved away from the contact producing side effects while at the same time preserving the relative strength of stimulation for the activated steered contact at that level. Fine tuning of the amplitude was then performed as needed in order to achieve optimal symptom control.

\section{Outcome Measures}

The number of required programming visits, patient's calls to the clinic, and parameter changes (changes in contacts, pulse width, or frequency) performed were the primary outcomes. Levodopa equivalent daily doses (LEDD) [6], Montreal Cognitive Assessment, and the Movement Disorders Unified PD Rating Scale (MDS-UPDRS) I-IV scores were recorded prior to surgery during a levodopa challenge (part III was performed off and on levodopa). Adverse events were recorded at each visit and at the end of the 3-month follow up. Side effects were considered significant if requiring changes in programming, either due to their negative impact on quality or life or if limiting further adjustments in stimulation and resulting in suboptimal control of symptoms. MDS-UPDRS III-IV and LEDD were also assessed at the end of 3 months. Battery consumption as measured by the physician's programmer was also recorded and expressed in terms of recharge time. All patients provided written consent, and the study was approved by our Research Ethics Board. 


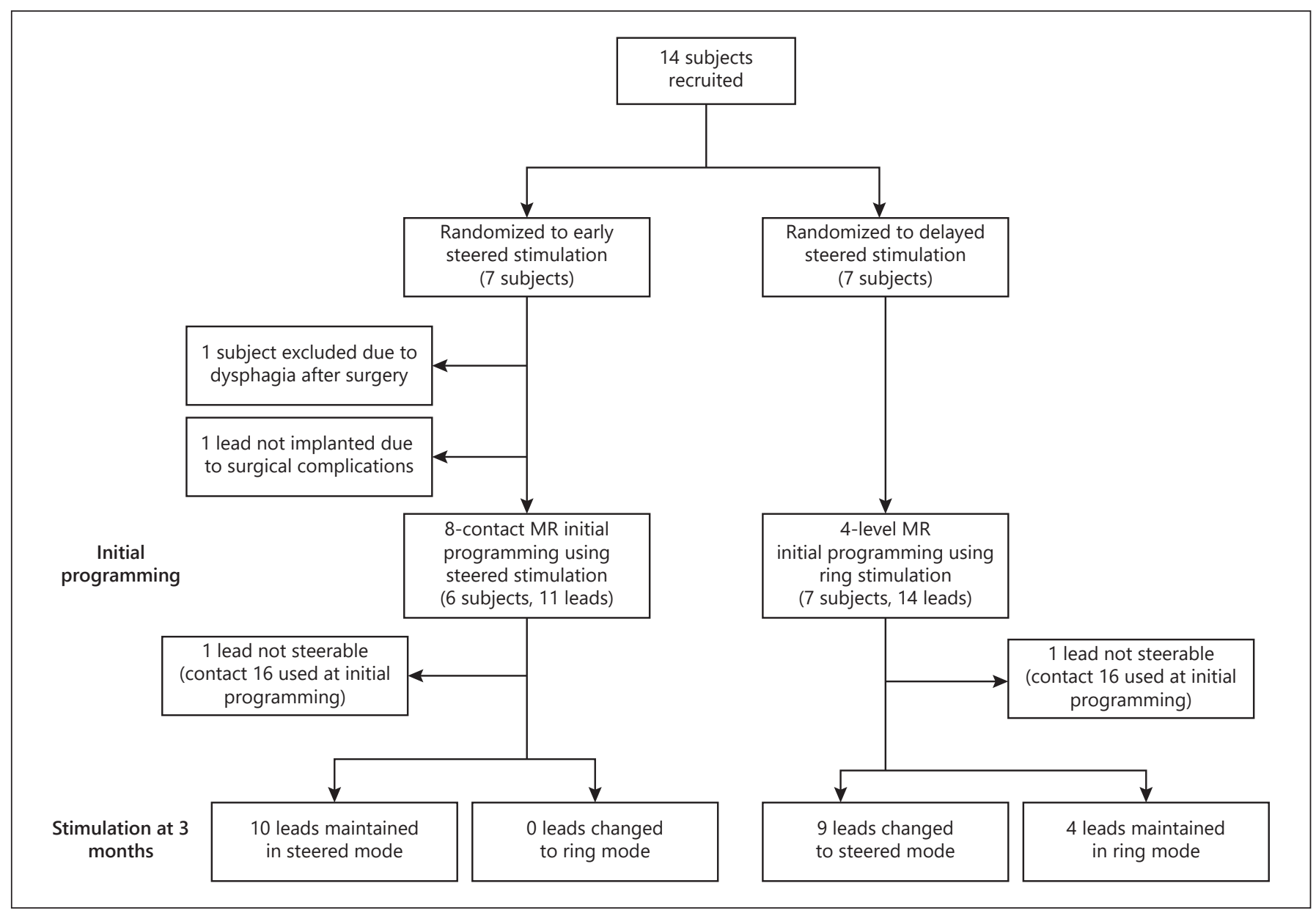

Fig. 2. Study flowchart. MR, monopolar review.

\section{Statistical Analysis}

The statistical analysis was performed using software package IBM SPSS Statistics ${ }^{\mathrm{TM}}$, version 23 (IBM, Armonk, NY, USA). Normality was assessed with the Shapiro-Wilk test. Baseline characteristics and outcomes were compared between groups by using independent sample $t$ and Mann-Whitney $U$ tests for normal and nonparametric continuous variables, respectively. The $U$ test was also used for ordinal variables. The Wilcoxon signed-ranks test was used for comparing outcomes in each group before and after surgery (MDS-UPDRS scores and LEDD). Additionally, the $t$ test and $U$ test were also used for comparison of change from baseline in scores between groups. The $\chi^{2}$ test was used to compare the proportion of contacts switched from one strategy to the other after 3 months. All tests were 2 -tailed with $\alpha=0.05$.

\section{Results}

No between-group differences in any of the demographic and clinical measures were present at baseline (shown in Table 1). There was 1 dropout due to surgical complications not related to stimulation (dysphagia requiring gastrostomy, attributed to a microlesion in association with premorbid cervical osteophytes) in a patient initially allocated to ESS. Another patient - also allocated to ESS - only underwent unilateral lead implantation due to poor compliance during the procedure.

Compared to baseline, DBS was equally successful in both groups, as indicated by the moderate reduction of MDS-UPDRS III off medication scores ( $p: 0.028$ and 0.046 for DSS and ESS, respectively) and LEDD reduction at 3-month evaluation ( $p: 0.028$ and 0.043 ). MDS-UPDRS IV scores were lower after 3 months, but this was not statistically different before and after surgery regardless of the stimulation strategy.

\section{Programming}

At the end of the first programming visit, all chosen levels were in a steerable part of the lead with the excep- 
Table 2. Contact selection and stimulation side effects for all patients

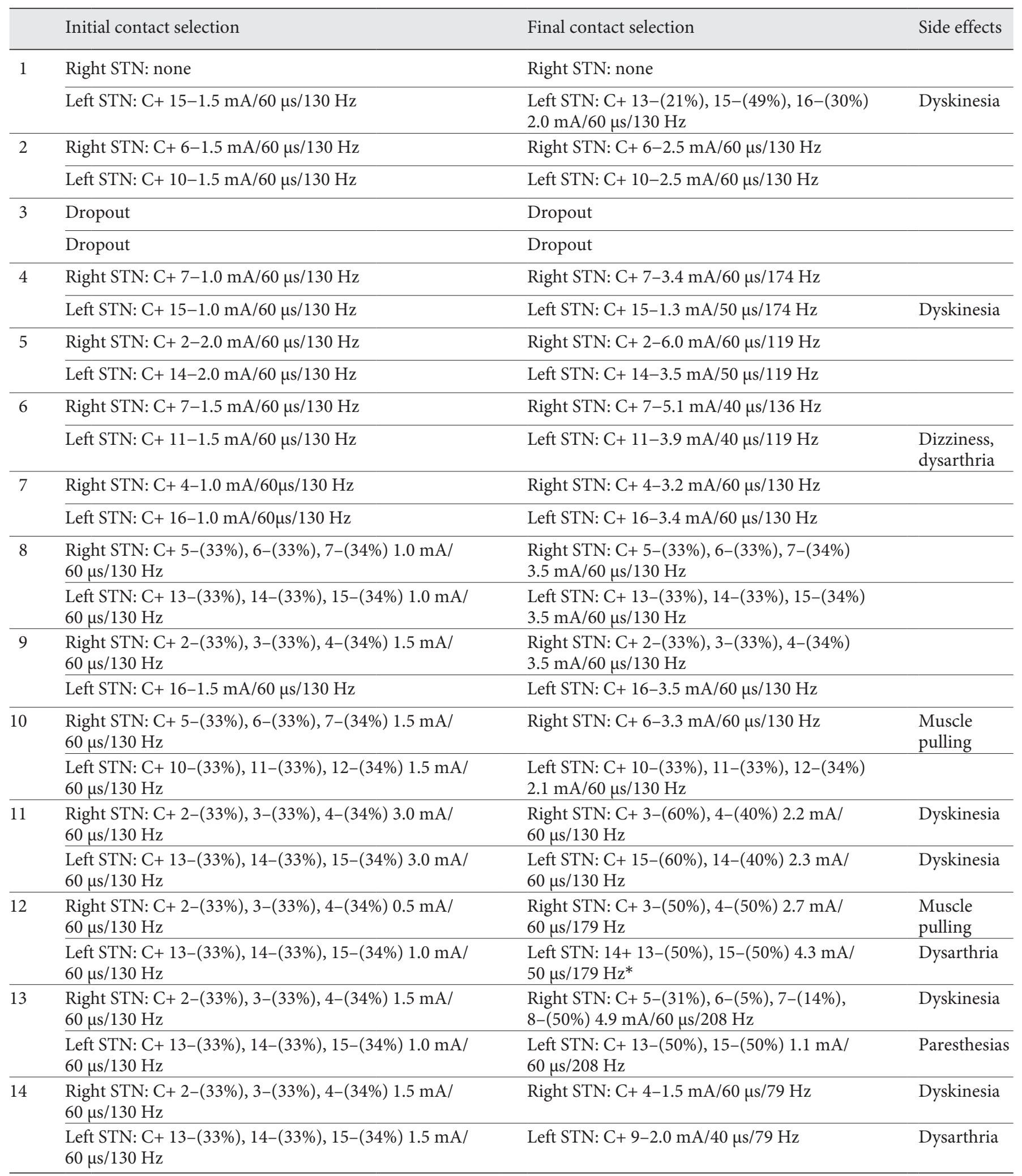

STN, subthalamic nucleus. * This configuration achieved better results than unipolar stimulation using contacts 13 or 15 for the control of dysarthria in this patient. 
tion of 2 leads (one per group) where the best level was the most dorsal contact (cases 7 and 9, see Table 2). Fourteen leads (13 of each steerable) could be evaluated in the DSS group, whereas 11 (10 of each steerable) were available for the ESS group (shown in Fig. 2).

During the programming phase, 9 leads (69.2\%) allocated to DSS were changed to steered. Reasons for change include capsular side effects in 3 electrodes, dyskinesia in 4 , and dysarthria in 2 electrodes. All side effects improved after steered stimulation.

Side effects in the ESS included 1 case of dizziness and dysarthria improved by reducing pulse width and 2 cases of dyskinesia (1 improved with activation the dorsal contact, and the other partially resolved with medication and amplitude reduction). No electrodes were changed from steered to "ring" stimulation ( $p$ : 0.001 vs. DDS).

There was no statistical difference when comparing all measures at 3 months after DBS between DSS and ESS (shown in Table 1). Initial and final contact selection and adverse events for all patients can be found in Table 2.

\section{Discussion}

Current programming paradigms rely on trial-and-error approaches which can be time consuming and burdensome. With the availability of steered leads, the number of available options for programming has exponentially increased, and the impact of having more contact configurations available on programming time and health care utilization needs to be considered when devising programming guidelines.

With this study, we aimed to compare the short-term outcomes of 2 initial programming paradigms; one where directionality was utilized "preemptively" (ESS) and another where directionality was used "as needed" (DSS). Our results show that the burden of both approaches on health care utilization (i.e., number of scheduled and unscheduled visits, phone calls, and stimulation changes) is similar, and having more contact configurations available did not translate into a higher number of stimulation adjustments or health care interactions before stimulation was considered satisfactory (e.g., fluctuations and dyskinesias under control), although there is a possibility that a difference might have been detected if the study included a higher number of patients (type II error). It is also important to note that the MR for ESS takes up to twice as long to complete [1].

Directional Deep Brain Stimulation

Programming for Parkinson's Disease
At the same time, the flexibility of having directionality as an option proved useful, as approximately $70 \%$ of electrodes programmed in ring mode initially were steered by the end of follow-up. The most common reasons for switching were capsular side effects, which is in keeping with rationale that steered stimulation can reduce spread of stimulation to the vicinity of the STN. Steered stimulation was also effective in managing stimulation-related dyskinesias (i.e., present in the off-medication state). Steered stimulation was attempted whenever reduction of stimulation amplitudes (current or pulse width) was not sufficient to control dyskinesias without worsening of the parkinsonism. In some patients, steering stimulation was used with the rationale that a better coverage of the STN might provide better control of symptoms at lower amplitudes, while in others, it was used to stimulate the dorsal contacts in an attempt to stimulate the pallido-fugal fibers in the zona incerta (as shown in Table 2). We did not assess precise electrode coordinates and orientation for this study and thus cannot guarantee that stimulation of the proposed structures was achieved. Furthermore, for the same reason, it is possible that there were differences between the 2 groups with respect to exact electrode location. How image-guided programming will inform the approach proposed in our study is presently unknown and will certainly be explored in the future. Of interest, no electrodes programmed to early directional stimulation had to be switched to ring mode. Finally, although the design of the current leads for steered stimulation is not perfect as they steer current only at the 2 middle levels [7], in our study, the vast majority of the chosen contacts $(>90 \%)$ were in a steerable region of the lead.

Our study is the first of its kind, but it has limitations. In particular, this study is open labeled, and placebo effect is possible. The MDS-UPDRS was scored by the unblinded programmer, but the assessor in each participant was the same for all visits for consistency of ratings. A bias to switching to steered stimulation could explain in part the prevalence of directional steering by the trial end. We have also recently shown that a more sophisticated approach to DBS (ESS in this study) can contribute to excessive expectations [4]. The short follow-up period may be another limitation, especially considering that the overall reduction in MDS-UPDRS III scores in both groups was less than usually expected after DBS (36.3 and 17.9\% for DSS and ESS, respectively, $p=0.18$ ). Switching stimulation from steered to ring mode was attempted to improve motor outcomes in some patients during the programming sessions, but in none of the cases better MDS- 
UPDRS III scores were achieved in the clinic when this was attempted. Finally, sample size is small and also complicated by the uneven number of explored leads between groups.

In conclusion, our study found that programming with ESS or DSS is equally effective in the short term. However, delayed steering is less time consuming as it involves a shorter initial programming visit, and steering might not be needed in a small proportion of patients. Taking these considerations into account, at the moment, we can recommend a level-by-level programming with a "ring mode" with further steering if needed during the course of programming.

\section{Statement of Ethics}

All patients gave their written informed consent to participate in this study. This research was conducted ethically in accordance with the World Medical Association Declaration of Helsinki. This study was approved by the University Health Network Research Ethics Board at University of Toronto (Reference No. 17-5707).

\section{Conflict of Interest Statement}

A.M.L. and A.F. received honoraria and research support from Boston Scientific. A.F. received honoraria from Abbott. R.M., D.S., R.P.M., Y.Y.P., and M.H. have nothing to declare. S.K. is a consul- tant for Medtronic. A.M.L. is a consultant for Boston Scientific, Medtronic, and Abbott. A.F. received honoraria for consultancies from Abbvie, Abbott, BrainLab, Boston Scientific, Chiesi Farmaceutici, Ipsen, Medtronic, Sunovion, and UCB; honoraria for participation in advisory boards from Abbvie, Boston Scientific, and Ipsen; research grants from Abbvie, Boston Scientific, Cummings Foundation, Dystonia Medical Research Foundation Canada, Michael J. Fox Foundation, Medtronic, University of Toronto, and Weston foundation.

\section{Funding Sources}

This study received funding from the Chair in Neuromodulation and Multidisciplinary Care at the University of Toronto and University Health Network (A.F.).

\section{Author Contributions}

Drs. Derrick Soh and Alfonso Fasano designed the study. Drs. Ricardo Maciel, Derrick Soh, Renato Munhoz, Alfonso Fasano, and Mrs. Yu-Yan Poon were involved in the organization of the research project. Drs. Ricardo Maciel, Suneil Kalia, Mojgan Hodaie, and Andres Lozano conducted the study, including patient recruitment and data collection. Dr. Ricardo Maciel was responsible for the statistical design of the study and data analysis. Dr. Alfonso Fasano provided statistical support and critique. Dr. Ricardo Maciel prepared the manuscript draft with important intellectual input from Drs. Derrick Soh and Alfonso Fasano. All authors approved the final manuscript.

\section{References}

1 Dembek TA, Reker P, Visser-Vandewalle V, Wirths J, Treuer H, Klehr M, et al. Directional DBS increases side-effect thresholds: a prospective, double-blind trial. Mov Disord. 2017;32(10):1380-8.

2 Shao MM, Liss A, Park YL, DiMarzio M, Prusik J, Hobson E, et al. Early experience with new generation deep brain stimulation leads in Parkinson's disease and essential tremor patients. Neuromodulation. 2019; 23(4):537-42.
3 Picillo M, Lozano AM, Kou N, Munhoz RP, Fasano A. Programming deep brain stimulation for Parkinson's disease: the Toronto western hospital algorithms. Brain Stimul. 2016;9(3):425-37.

4 Soh D, ten Brinke TR, Lozano AM, Fasano A. Novel deep brain stimulation technologies for Parkinson's disease: more expectations, more frustrations? Mov Disord Clin Pract. 2019; $7(1): 113-4$
5 Munhoz RP, Picillo M, Fox SH, Bruno V, Panisset M, Honey CR, et al. Eligibility criteria for deep brain stimulation in Parkinson's disease, tremor, and dystonia. Can J Neurol Sci. 2016;43(04):462-71.

6 Tomlinson CL, Stowe R, Patel S, Rick C, Gray $\mathrm{R}$, Clarke CE. Systematic review of levodopa dose equivalency reporting in Parkinson's disease. Mov Disord. 2010;25(15):2649-53.

7 Fasano A, Lozano AM, Cubo E. New neurosurgical approaches for tremor and Parkinson's disease. Curr Opin Neurol. 2017;30(4): $435-46$. 